\title{
ESTUDIO FITOQUIMICO DE EXTRACTOS DE Alternanthera sessilis L.Y SU EVALUACIÓN PARA EL TRATAMIENTO DE LA PSORIASIS
}

\author{
Alternanthera sessilis L. EXTRACTS: PHYTOCHEMICAL AND ANTIPSORIATIC \\ EVALUATION
}

\section{PHYTOCHEMICAL AND ANTIPSORIATIC EVALUATION OF Alternanthera sessilis L. EXTRACTS}

\author{
SALAS OLIVET, EVA ${ }^{1 *}$ ING.; GARCÍA, SIMÓN GASTÓN ${ }^{1}$ M.Sc., CUÉLLAR \\ CUÉLLAR, ARMANDO ${ }^{1}$ Ph.D., SCULL LIZAMA, RAMÓN M ${ }^{1}$.Sc.
} ${ }^{1}$ Departamento de Farmacia, Instituto de Farmacia y Alimentos, Universidad de
la Habana, Cuba.

${ }^{*}$ Correspondencia: evaso@inmail24.com

Recibido: 19 -12- 2010; Aprobado: 7 -05- 2011.

\section{Resumen}

Alternanthera sessilis L. (Amaranthaceae), es una planta rastrera muy común en Cuba que se utiliza tradicionalmente para afecciones gastro intestinales, así como astringente, abortiva y diurética. Nuestro grupo de trabajo comenzó el estudio para el tratamiento de la psoriasis teniendo en cuenta la referencia de su uso tradicional para la urticaria. Se realiza un estudio Fitoquímico que contempla la determinación de algunos parámetros farmacognósticos y extractos con disolventes de polaridad creciente, aislándose varios compuestos tipo carotenoides, triterpenoides y glicósidos, informándose los métodos de trabajo utilizados y sus resultados más importantes. El extracto acuoso se evalúa en ratones, comparando con Coriodermina, método utilizado por el Centro de Investigaciones Placentarias de Cuba, demostrando que el mismo posee un afecto ligeramente inferior que la Coriodermina en el incremento del espesor de la epidermis. Estos resultados son novedosos para la planta y en estos momentos se evalúan los productos aislados para determinar su estructura química y su contribución al efecto encontrado experimentalmente.

Palabras clave: Alternanthera sessilis L, efecto antipsoriático, Cuba.

\section{Abastract}

Alternanthera sessilis L. (Amaranthaceae), is a creeping herb that wildly grows in Cuba. Traditionally is used mainly for the treatment of gastric illnesses and as astringent, abortive and diuretic. Our group began the present study because of the use for the treatment of urticarial. A phytochemical study was carried out with the determination of some pharmacognostic assays that include extraction 
with solvents of increasing polarity that allowed the isolation of carotenoids, triterpenoides and glycosides, now under structure elucidation. The methods used are described. Aqueous extract was evaluated in mice for the possible anti psoriatic activity in comparison with Coriodermine measuring the increase on the epidermis layer on the tail of the experimental animals. Those results are reported for the first time for the plant that produces a positive result for the treatment of this affection of the skin.

Key words: Alternanthera sessilis (L), anti psoriatic effect, Cuba.

\section{Introducción}

Alternanthera sessilis $(\mathrm{L}) \mathrm{R}$. Br es una planta rastrera de la familia Amarantácea (Fig. 1), que suele encontrarse en las costas y terrenos arenosos en Cuba. (ROIG, 1988). El mismo autor la describe como una hierba pelosa, ramificada, con las ramas postradas, hojas opuestas, enteras, espatuladas, ovadas hasta ovales. Flores perfectas en densas cabezuelas sésiles color blanquecino mate y semillas lenticulares lisas.

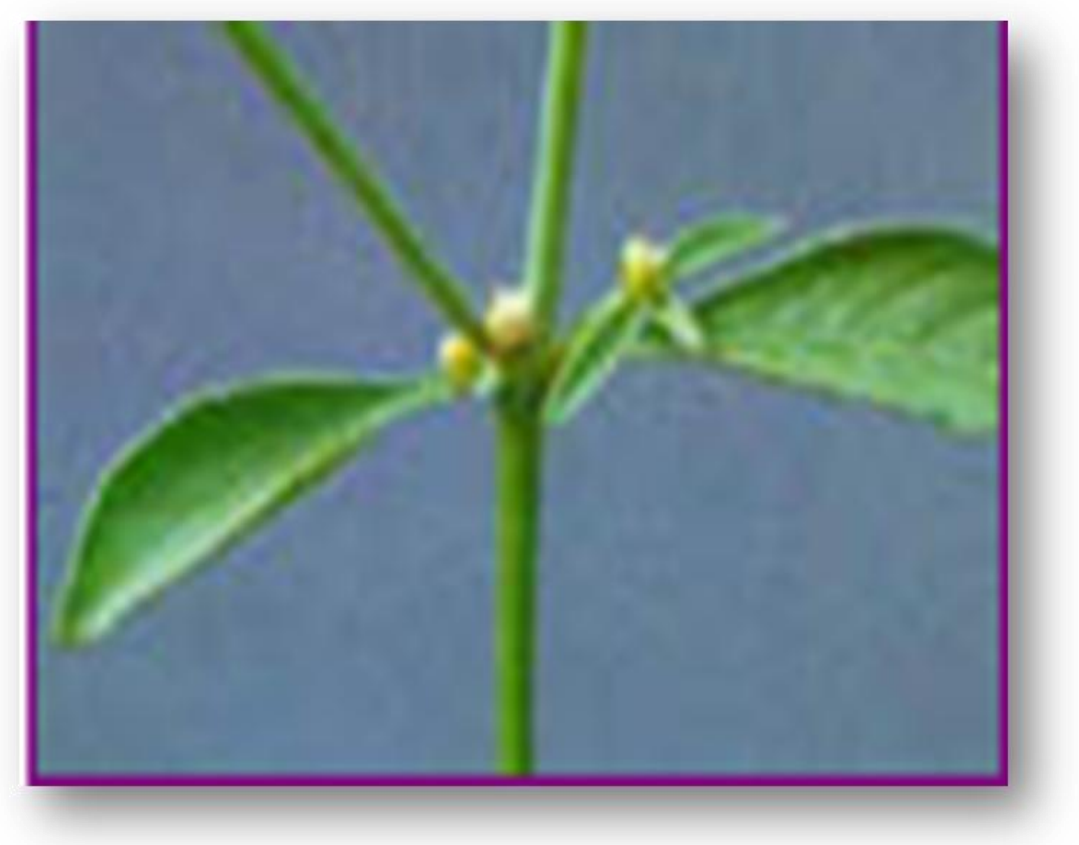

Figura1. Imagen de Alternanthera sessilis $L$

En cuanto a estudios realizados con la misma, se describe el aislamiento y caracterización de sitosteroles (SAHU et al., 1993) quien aisló también de las hojas secas de la planta glicósidos flavonoides. Además, BLUNDEN (1999) resume la familia Amarantácea entre ella Alternanthera sessilis $L$. como productora de Betaleinas. Por otra parte, RAGASA et al. (2002) reportan que los extractos de cloroformo a partir de las hojas secas contienen diferentes iononas. 
Desde el punto de vista comercial como medicinal, (DEUG et al., 2006) presenta una patente de una combinación de plantas en China con 16 diferentes especies que incluye Alternathera sessilis $L$. La preparación se enriquece con potasio y se utiliza para el tratamiento de la hipertensión arterial. Mientras (MAYANK, 2006) la incorpora en un preparado de la medicina tradicional Ayurveda de la India para el tratamiento de Rickettsia y Marasmius $\mathrm{NIE}$, en 2007 patenta la incorporación de 7 plantas que se usan en China para tratar la alopecia entre las que se encuentra la Alternanthera sessilis $L$. Mientras (WU, 2007) la incorpora a un vinagre medicinal preparado en China para uso oral. Otra inventiva fue desarrollada por $(X U, 2008)$ la que comprende una simple preparación por decocción de una formulación compuesta por Alternanthera sessilis $L$. y otras 5 plantas para el tratamiento de la gonorrea en mujeres, con bajos efectos colaterales y bajos costos.

Recientemente UNMI et al. (2009) desarrolla un estudio con 9 plantas medicinales donde una es Alternanthera sessilis $L$., las que presentan actividad antibacteriana frente a Escherichia coli Por su parte, (CHANDRIKA et al., 2006) determinó que la Alternanthera sessilis $L$. aporta de 14 miligramos a un gramo de beta caroteno, precursor de la vitamina $A$, por lo que puede utilizarse como comestible y con fines nutricionales tanto para el hombre como para los animales.

MOODLEY et al. (2007) se refieren a Alternanthera sessilis L. como una planta que se utiliza para desintoxicar suelos y aguas residuales de metales pesados tales como $\mathrm{Cr}, \mathrm{Pb}$ y Ni por su capacidad de absorber y acumular los mismos. El poder de acumulación está en el orden del 15\% además de absorber $\mathrm{Fe}, \mathrm{Ca}, \mathrm{Zn}, \mathrm{Cd}, \mathrm{As}, \mathrm{Cu}, \mathrm{N}, \mathrm{P}$ y K por lo que es una planta que debe establecerse su potencial tóxico antes de su utilización en humanos o en animales.

\section{Materiales y métodos}

\section{Estudio Farmacognóstico}

Material Vegetal. El material vegetal empleado en el presente estudio se identificó en el Jardín Botánico Nacional de Cuba con el número de herbario HFC 85590 (HAJB); como la especie Alternanathera sessilis L. la que se colectó en San Agustín La Lisa, Provincia Ciudad de la Habana, Cuba. Los ejemplares seleccionados de Alternanathera sessilis $L$. se procesan como planta entera, utilizando tijeras de corte para su división.

Los estudios Farmacognósticos se realizaron de acuerdo a (WHO/PHARM/ 80.50). Se evaluaron los siguientes parámetros de calidad de la materia prima vegetal: Secado en diferentes condiciones, determinación de humedad 
residual, determinación de cenizas, sólidos solubles extraíbles en diferentes disolventes de polaridad creciente y un Tamizaje Fitoquímico general de acuerdo a (CUÉLLAR y GONZÁLEZ, 2010)

Extracción: El material vegetal se somete a un proceso de extracción por disolventes sucesivos utilizando hexano, cloroformo, etanol y agua sobre el mismo material vegetal, de acuerdo con (HOSTETTMANN et al., 1986). En el presente trabajo sólo se informará lo referente al extracto acuoso en cuanto a su evaluación del posible efecto anti psoriático ya que es el utilizado de forma tradicional.

\section{Ensayo del efecto anti psoriático de un extracto acuoso de Alternanthera sessilis $L$.}

Se determinó el efecto anti psoriático de éste producto mediante el ensayo que analiza la medida del grosor de la epidermis luego de la aplicación del producto a evaluar. (MARTOJA et al., 1970)

\section{Modelo Animal}

Animales: Para la experiencia se emplearon ratones machos NMRI, procedentes del CENPALAB (Centro Nacional para la Producción de Animales de Laboratorio) los cuales se mantuvieron en cuarentena. Todos los ratones tenían su certificado de calidad que garantizaba la salud de los mismos así como que se encontraban aptos para efectuar este tipo de ensayo.

El peso de los ratones variaba de 24-30 g. El acceso al agua y a la comida fue ad libitum. Las condiciones de luz y humedad fueron las descritas para la especie. El tratamiento se aplica de forma tópica en la cola del animal durante 21 días (sin incluir los sábados y domingos) de la forma siguiente:

Se retira el guante de la mano con la que le aplicará el tratamiento al animal. El animal se toma por la cola y se frota el producto en la cola durante 15 segundos. Se lavan las manos con agua y jabón entre grupos de tratamiento. Finalmente transcurrido $72 \mathrm{~h}$ horas de concluido el tratamiento se sacrifican los animales mediante tracción de las vértebras cervicales.

La distribución de los animales en los grupos fue al azar y se sub agruparon según se expresa en la Tabla 1.

Las muestras se recogieron de la manera siguiente:

- Se realizó un corte con el bisturí alrededor de la base de la cola y después a lo largo de la línea media de ésta. 
- -Se desprendió cuidadosamente la unión entre las intersecciones de los cortes en la base de la cola y se tiró hacia abajo (retrayéndola) hasta sacar la cola completamente.

- Se cortó un fragmento de $1 \mathrm{~cm}$ aproximadamente correspondientes a la parte de la base de la cola.

- Las muestras de cada grupo se envolvieron en gasa, colocándole un papel para identificarlos.

- La gasa se amarró con un hilo o cordel.

- Las gasas se colocaron en un frasco con formol 10\% durante 48 a 72 horas.

- Se entregaron al patólogo para su procesamiento y lectura

Tabla 1. Grupos que se sometieron a ensayo

\begin{tabular}{|r|l|}
\hline \multicolumn{1}{|c|}{ Grupo } & \multicolumn{1}{|c|}{ Tratamiento en los grupos } \\
\hline I & Extracto acuoso de Alternanthera sessilis L. \\
\hline II & Coriodermina \\
\hline III & Placebo. \\
\hline IV & Sin Tratamiento \\
\end{tabular}

Cálculos estadísticos. Se calcularon la media y la desviación de cada medición realizada en los diferentes grupos, y a continuación se aplicó un Análisis de Varianza de una Vía de clasificación y a posterior la prueba de Student Newman Keuls $(p<0.05)$.

Dosis y duración del ensayo. Se utilizó la vía tópica para administrar los diferentes compuestos en la cola del animal durante 21 días (sin incluir los sábados y domingos). El ensayo tuvo una duración de 26 días en total.

Sacrificio de los animales. Los animales fueron sacrificados mediante dislocación cervical, cumpliendo con los procedimientos de Refinamiento, o sea evitar el dolor y el sufrimiento de los mismos.

\section{Resultados y discusión}

Estudio del secado: El estudio del secado se realiza en dos posibles variantes con tres réplicas en cada ensayo para valorar la mejor opción en cuanto a 
tiempo de duración y eficiencia en la pérdida de agua, ya que de ellos depende la calidad de la droga a trabajar En la Tabla 2 se presentan los resultados obtenidos donde tal y como se observa, el mejor método fue el de secado artificial (estufa a $45^{\circ} \mathrm{C}$ ) ya que se seca el material vegetal en el menor tiempo con una pérdida apreciable de agua, el $82 \%$. Los resultados del contenido de humedad residual promedio de tres determinaciones, fue de $11.8 \%$ que aunque algo elevado se encuentra en el rango normado, no mayor de $12 \%$ para la mayoría de las plantas medicinales.

Tabla 2. Resultados de la evaluación de los métodos de secado

\begin{tabular}{|l|l|l|}
\hline Métodos Parámetro & $\begin{array}{c}\text { Tiempo } \\
\text { (días) }\end{array}$ & $\begin{array}{c}\text { Pérdida de peso } \\
\text { (\%) X } \mathbf{~ D E}\end{array}$ \\
\hline Sombra & 15 & $64,48 \pm 0,06$ \\
\hline Estufa & 3 & $82,02 \pm 1,00$ \\
\hline
\end{tabular}

Índices numéricos para la calidad de la materia prima: Se realizó la determinación de algunos parámetros de calidad de la droga después de secada a la estufa, los que se relacionan a continuación con los siguientes resultados:

Las cenizas totales tuvieron un valor promedio de $5 \%$ en el límite de muchas de las plantas medicinales comerciales pero al realizar la determinación de las cenizas solubles en agua el valor fue de $2,46 \%$ por lo que la diferencia es de $2,54 \%$ respecto a las totales lo cual asegura según lo establecido al estar por debajo del $3 \%$ que no hay riesgos de tener grandes cantidades de metales pesados que puedan suministrar algún tipo de toxicidad a los extractos preparados Esto se confirma por el valor de las cenizas insolubles en ácido que fue de un $1,04 \%$ lo que asegura valores de composición inorgánica aceptables para las plantas medicinales sin la presencia de metales que puedan aportar toxicidad por encima de los límites aceptados.

Esto se corrobora al determinar la composición inorgánica general en el Centro de investigaciones para la industria minero metalúrgica mediante el siguiente Procedimiento: Los metales As y Se por digestión ácida y determinación final por ICP. El resto de los metales por calcinación de las muestras, digestión ácida y determinación final por ICP. La materia orgánica se calcinó a $900^{\circ} \mathrm{C}$ y la humedad se obtuvo por el método gravimétrico. Los resultados se observan en la Tabla 3. 
Tabla 3. Contenido en \% de la composición inorgánica de Alternanathera sessilis $L$.

\begin{tabular}{|c|c|c|c|c|}
\hline Muestra & $\mathbf{C r}$ & $\mathbf{P b}$ & $\mathbf{N i}$ & $\mathbf{M g}$ \\
\hline ESO-M-1 & $<5,0 \times 10^{-5}$ & $7,99 \times 10^{-3}$ & $3,81 \times 10^{-4}$ & 0,72 \\
\hline & & & & \\
\hline & $\mathrm{Fe}$ & $\mathrm{Zn}$ & $\mathrm{Cd}$ & $\mathrm{As}$ \\
\hline ESO-M-1 & $2,4 \times 10^{-2}$ & $3,4 \times 10^{-3}$ & $6,1 \times 10^{-4}$ & $<2,0 \times 10^{-4}$ \\
\hline & & & & \\
\hline & $\mathrm{Mn}$ & $\mathrm{Se}$ & $\mathrm{Cu}$ & $\mathrm{Co}$ \\
\hline ESO-M-1 & $4,0 \times 10^{-3}$ & $1,0 \times 10^{-4}$ & $4,8 \times 10^{-3}$ & $5,2 \times 10^{-5}$ \\
\hline & & & & \\
\hline & $\mathrm{Al}$ & $\mathrm{V}$ & $\mathrm{Ti}$ & $\mathrm{Mo}$ \\
\hline ESO-M-1 & 0,13 & $1,9 \times 10^{-3}$ & $2,4 \times 10^{-3}$ & $<5,0 \times 10^{-5}$ \\
\hline & & & & \\
\hline ESO-M-1 & Mat. orgánica & $\mathrm{H}_{2} \mathrm{O}$ & & \\
\hline & 86,95 & 11,67 & & \\
\hline
\end{tabular}

Se determinaron además los sólidos solubles (extractivos) en los 4 disolventes de extracción, siendo los resultados los siguientes: Hexano (1,3\%), cloroformo ( $1 \%)$, etanol $(4,7 \%)$ y agua $(8 \%)$ todos referidos al peso de planta de partida.

El tamizaje Fitoquímico sugirió la presencia mayoritaria para el extracto de hexano de carotenoides y esteroles, para el cloroformo triterpenoides, para el alcohol compuestos fenólicos y glicósidos y para el agua compuestos reductores, compuestos fenólicos y/o taninos y saponinas.

El extracto acuoso sometido a la evaluación anti psoriática, aportó los resultados que se exponen en la Tabla 4.

Tabla 4. Resultados del ensayo del efecto anti psoriático del extracto acuoso de Alternanthera sessilis $L$.

\begin{tabular}{|l|l|}
\hline \multicolumn{1}{|c|}{ Grupo } & $\begin{array}{c}\text { Medida del grosor de la } \\
\text { epidermis }(\boldsymbol{\mu}) . \\
(\mathbf{X} \pm \mathrm{DS})\end{array}$ \\
\hline I .Alternanthera sessilis & $19.98 \pm 2.98 \mathrm{a}$ \\
\hline II. Coriodermina & $18.53 \pm 2.20 \mathrm{~b}$ \\
\hline III. Placebo. & $22.72 \pm 2.78 \mathrm{c}$ \\
\hline IV. Sin Tratamiento & $21.98 \pm 1.38 \mathrm{c}$ \\
\hline
\end{tabular}

a,b,c, significación estadística $(p<0.05)$ 
El extracto de Alternanthera sessilis $\mathrm{L}$ se considera que tiene efecto anti psoriático ya que existen diferencias significativas con el placebo y el grupo no tratado y no difiere con respecto a la Coriodermina tomada a manera de control positivo. Esto se traduce en que el grosor del epitelio deberá ser menor en los tratados con los productos respecto a los grupos no tratados y del placebo.

Como se puede apreciar los grupos no tratados y el placebo no diferían entre sí, sin embargo los que fueron tratados con la Coriodermina y el extracto acuoso de Alternanthera sessilis $L$. difieren de estos y entre ellos.

Estos resultados indican que el extracto acuoso de Alternanthera sessilis $L$ se considera que tiene efecto anti psoriático dado a que como se puede observar existen diferencias significativas con el placebo y el grupo no tratado pero difiere de la Coriodermina tomada a manera de control positivo, lo cual indica que existe presencia de efecto antipsoriático pero en un grado menor que la Coriodermina utilizada como control positivo.

\section{Referencias}

BLUNDEN, G.; YANG, M.; JANICSAK, G.; CARABOT, A. 1999. Betain distribution in Amaranthaceae. Biochemical Systematic and Ecology 27(1):8792.

CUÉLLAR, A.; GONZÁLEZ, J. 2010. Obtención del glucósido flavonoide Gossypitrina de los pétalos de las flores de Talipariti elatum S. W. y evaluación de su posible efecto antioxidante. RECIA 2 (2): 338-347.

CHANDRIKA, U.; SVANBERB, U.; JANZ, Z. 2006. In vitro accessibility of beta carotene from cooked Srilanka green leafy vegetable and their estimated contribution to Vit A requirements. J. of the Science of Food and Agriculture 88 (1):54-61.

DENG, J; GANG, W. 2006. Patent in China. Application CN 2005-10072497 20050522.

hOSTETTMANN, K.; HOSTETTMANN, A.; MARSON, A. 1986 Preparative chromatography Techniques. Cap 2. Sample preparation and purification. Editorial Springer-Verlag.

MARTOJA, R.; MARTOJA, M. 1970. Técnicas de Histología Animal. Editorial Toray-Masson, S.A Barcelona, España.

MAYANK, A. 2006. Patent in India. Application IN 2004 DE 189320040930

MOODLEY, K.; BAIJAATH, H.; SAUTHWAY, F.; MAHARAJ, S.; CHITTY. S. 2007. Determination of $\mathrm{Cr}, \mathrm{Pb}$ and $\mathrm{Ni}$ in water, sludge and plants from settling ponds of a sewage treatment work. Durban S. African Water S.A. 33(5):923928. 
NIE, Y. 2007. Patent in China. Application CN 2006-10070014 20061028

RAGASA, C.; TREMOR, N.; RIDEOUT, J. 2002. Ionone derivatives from Alternanthera sessilis. J. of Asian Natur. Prod. Research 4(2):109-115.

ROIG, J.T. 1988. Plantas Aromáticas, Venenosas y Medicinales de Cuba. Editorial Científico Técnica, Habana Cuba.

SAHU, B.R.; CHAKRABARTY. A. 1993. A flavone glycoside Robinitine from Alternanthera sessilis. Journal of Chemistry 5(4):1148-1150.

UMNI, B.; BORAH, A.; WANN, S.B.; SINGH, H.A. BASABRANI, B. 2009. Phytochemical and antibacterial study of traditional medicinal plants of North West India on E. coli. Asian J. of Experimental Sciences 23(1):103-108.

WU, S. 2007. Patent in China. Application CN 2005-10015356 20051010

XU, P. 2008. Patent in China. Application CN - 1013024320080610 